\title{
A Triangulation of Moduli Space from Light-Cone String Theory
}

\author{
Steven B. Giddings ${ }^{1, \star}$ and Scott A. Wolpert ${ }^{2, \star \star}$ \\ ${ }^{1}$ Joseph Henry Laboratories, Princeton University, Princeton, NJ 08544, USA \\ ${ }^{2}$ Department of Mathematics, University of Maryland, College Park, MD 20742, USA
}

\begin{abstract}
We show that scattering diagrams for closed strings in light-cone string theory provide a single cover of the moduli space of Riemann surfaces.
\end{abstract}

\begin{abstract}
Since the early days of string theory physicists have known that modular invariance, or more accurately, invariance under the mapping class group, plays a fundamental role in the theory. Modular invariance is especially relevant to such consistency issues as unitarity and finiteness. In the Polyakov approach [1] to string theory modular invariance is seen directly: the integrand in correlation functions or the partition function is modular invariant in the critical dimension, and the integration region is taken to be one copy of the fundamental domain of the mapping class group, or equivalently moduli space. This approach to string theory is very elegant, but such basic properties as unitary are not manifest.

There has traditionally been another way of approaching string theory, and that is the interacting string picture as formulated in the light-cone gauge [2]. In this approach Lorentz invariance is not manifest, but unitarity is apparent since we work only with physical states. However, it has not been proven that the light-cone picture is equivalent to the Polyakov picture. One of the steps in this proof is settling the question whether the light-cone formalism reproduces the integration over a single copy of the moduli space for a Riemann surface of arbitrary genus. Some physicists have implicitly assumed that the answer to this question is yes. In fact, it is hard to imagine that the Polyakov theory is correct if it isn't equivalent to the light-cone formulation ${ }^{1}$ since the light-cone formulation manifests essential properties like unitarity. If the light-cone formulation didn't reproduce the integration over moduli space we would have to add or subtract by hand the integration over the rest of moduli space; such tinkering could easily ruin the nice properties of the theory.
\end{abstract}

* Supported in part by a National Science Foundation Graduate Fellowship

$\star \star$ Supported in part by a National Science Foundation grant

${ }^{1}$ We thank S. Mandelstam for a discussion on this point 
At first sight, the statement that light-cone diagrams give a one-to-one cover of moduli space is not at all obvious. One reason for this is that moduli space is topologically complicated (its Euler character is [3] $\zeta(1-2 g) \sim \frac{(-)^{g}(2 g-1) !}{2^{2 g-1} \pi^{2 g}}$ ), yet in the light-cone picture we are representing it as one topologically trivial region with identifications on its boundary (a cell in mathematical language). We have a single cell since, roughly speaking, for a given topology there is only one light-cone diagram, and we can thus pass from any point in moduli space to any other by a continuous deformation of this diagram that avoids the boundary configurations, i.e. those in which interaction points merge or go to infinity. It is not easy to see precisely what the identifications on the boundary of the cell look like; they are implicit in the construction of the light-cone diagrams but are necessarily not simple since the topological complexity of the moduli space must manifest itself in these identifications.

Therefore the statement that light-cone diagrams cover moduli space is nontrivial. From the point of view of string theory it is of course plausible, and in fact the open string field theory discovered by Witten [4] gives an example of how something invented purely within the context of string theory was later found to give a (previously known [5]) cell decomposition of moduli space [6]. As we will see in this paper, the light-cone formalism for the closed string does indeed provide a single cover of moduli space. Further, this cell decomposition appears to be nicely related the complex structure of the moduli space.

We begin by examining a simple light-cone diagram; generic diagrams are shown in Fig. 1a and b. A diagram consists of flat tubes corresponding to free propagation of strings; at the interaction points there are curvature singularities. These correspond to the type of interaction shown in Fig. 2. The intermediate strings are allowed to twist by some angle between 0 and $2 \pi$ before rejoining; integration over this angle gives the projection onto states satisfying $L_{0}-\bar{L}_{0}=0$, where for $n$ an integer $L_{n}, \bar{L}_{n}$ denote the generators of the Virasoro algebra. In calculating scattering amplitudes in the light-cone gauge the circumference $2 \pi \alpha$ of a string is taken to be proportional to the component $P^{+}$of the total momentum of the string. The parameters that characterize such a diagram with $n$ external states and $g$ loops are then the momenta $\alpha_{i}, \alpha_{I}$ (here $i=1, \ldots, n$ and $I=1, \ldots, g$ ), the twist angles $\theta_{\beta}(\beta=1, \ldots, 3 g+n-3)$, and the interaction times $\tau_{a}(a=1, \ldots, 2 g+n-3)$. In the computation of a scattering amplitude the external momenta are fixed and the internal variables are integrated over, giving a total of $6 g+2 n-6$ parameters that label a diagram with $g$ loops and $n$ external states of fixed momenta. Specification of a flat metric with isolated curvature singularities on the diagram specifies a conformal structure ${ }^{2}$ on a Riemann surface of genus $g$ with $n$ punctures; the variables $(\alpha, \theta, \tau)$ are the modular parameters labelling the conformal structure. The question is whether we obtain each conformal structure from such a diagram, and whether there is only one such light-cone diagram that represents each conformal structure.

\footnotetext{
${ }^{2}$ Recall that a conformal structure on a topological surface is a metric defined modulo Weyl rescalings
} 


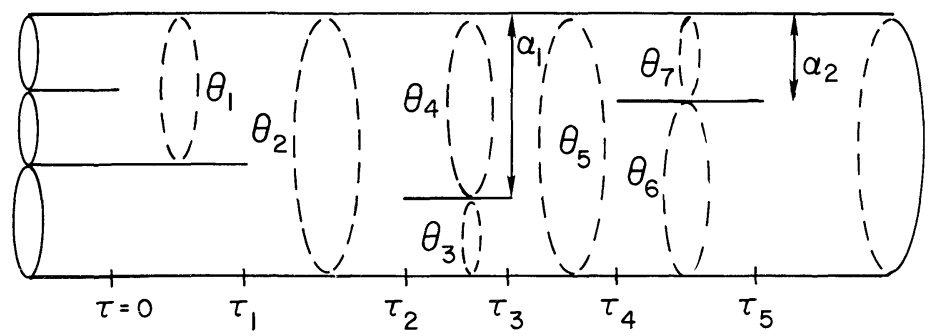

(a)

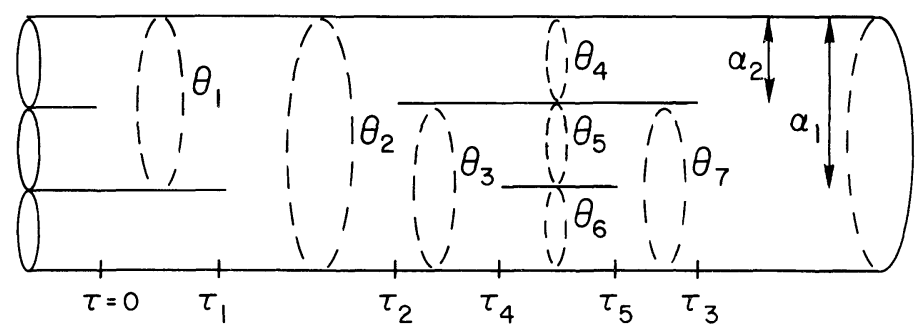

(b)

Fig. 1a and b. An example of a light-cone scattering process. Each internal tube is allowed to rotate by an angle $\theta_{\beta}$ between 0 and $2 \pi$ before undergoing another interaction; this is indicated by the dotted lines. The interaction times $\tau_{a}$ are defined up to an overall shift; we can use the the shift to set the first interaction time to $\tau=0$. Tubes corresponding to external strings are infinite in length. The radii of the tubes are the parameters $\alpha_{i}, \alpha_{I}$. In $\mathbf{a}$ and $\mathbf{b}$ are shown diagrams corresponding to different orderings of the times $\tau_{a}$

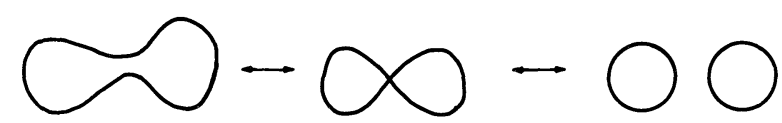

Fig. 2. The basic closed string interaction

The generic light-cone diagram is characterized by having only simple interactions: either a string interacts and forms two strings or two strings interact to form a single string. Such diagrams will henceforth be called simple. By contrast, examples will show that the combinatorics of a non-simple diagram can be rather complicated; once again, these correspond to boundaries of the top dimensional cell.

The point of this note is that the light-cone diagram is equivalent to the existence of an abelian differential (or meromorphic one-form) $\omega$ with specified residues (whose sum is zero) and pure imaginary periods. To see that a diagram indeed determines a differential it is easiest to consider the examples. For each example and in general we find that the natural abelian differential $d w$ on the world-sheet is the restriction of an abelian differential defined on a planar diagram from which the light-cone diagram can be obtained by identification of boundary segments. Since gluing is a local construction it is enough to discuss single 
interactions. The purpose of the following examples is to demonstrate the validity of the gluing and the existence of the abelian differential, and also to illustrate some of the non-trivial diagrams that can arise.

Example 1. (The simple interaction) Start with a horizontal strip in the $w$ plane with two slits (see Fig. 3a). For all examples the edges labeled by the same letter $\left(A, A^{\prime}\right.$, etc.) are to be identified by a vertical translation. The slit disc neighborhoods of $a$ and $b$ are identified to form a neighborhood of the interaction point. Define local coordinates $z_{a}=(w-a)^{1 / 2}, \quad 0<\arg \left(z_{a}\right) \leqq \pi, \quad z_{b}=(w-b)^{1 / 2}$, $\pi<\arg \left(z_{b}\right) \leqq 2 \pi$. A coordinate $z$ is defined on the glued surface by its restrictions $z_{a}$ and $z_{b}$ to half disc neighborhoods of the identified point $a \equiv b$. In particular $z_{a}^{2}=(w-a), 2 z_{a} d z_{a}=d w, z_{b}^{2}=(w-b), 2 z_{b} d z_{b}=d w$, and thus $2 z d z$ is transformed into the differential $d w$ by the change of coordinates.

Example 2. (2 incoming, 3 outgoing strings) see Fig. $3 \mathrm{~b}$. First slit neighborhoods of $b$ and $d$ are glued and then slit neighborhoods of $a, c$, and $e$ are glued. Let $z_{a}, z_{b}, \ldots$ be a coordinate for a slit disc at $a, b, \ldots$. Define $z_{b}=(w-b)^{1 / 2},-\frac{\pi}{2}<\arg \left(z_{b}\right) \leqq \frac{\pi}{2}$, $z_{d}=(w-d)^{1 / 2}, \frac{\pi}{2}<\arg \left(z_{d}\right) \leqq \frac{3 \pi}{2}$, and thus $z_{b}$ and $z_{d}$ define a coordinate $z_{1}$ on the glued discs. Similarly define $z_{a}=(w-a)^{1 / 3}, 0<\arg \left(z_{a}\right) \leqq \frac{2 \pi}{3}, \quad z_{e}=(w-e)^{1 / 3}$, $\frac{2 \pi}{3}<\arg \left(z_{e}\right) \leqq \frac{4 \pi}{3}, z_{c}=(w-c)^{1 / 3}, \frac{4 \pi}{3}<\arg \left(z_{c}\right) \leqq 2 \pi$ and thus $z_{a}, z_{e}$, and $z_{c}$ define a coordinate $z_{2}$ on the glued discs. Again $2 z_{1} d z_{1}$ and $3 z_{2}^{2} d z_{2}$ are transformed into the differential $d w$ by the change of coordinates.

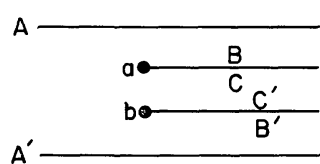

(a)

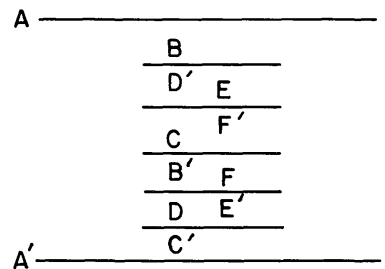

(c)

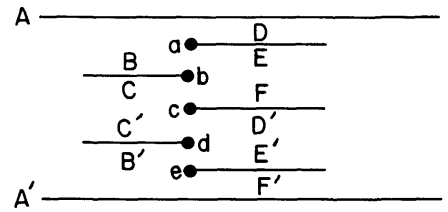

(b)

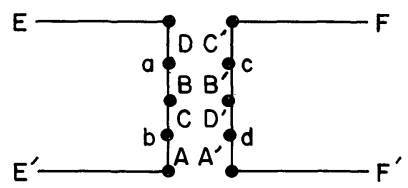

(d)

Fig. 3a-d. Planar regions from which light-cone diagrams can be obtained by identification of marked edges. In each case we identify the slit edges $A$ with $A^{\prime}, B$ with $B^{\prime}$, etc. In a is the simple interaction; in $\mathbf{b}$ a situation with two incoming and three outgoing strings, in $\mathbf{c}$ an example of nontrivial combinatorics, and in $\mathbf{d}$ a time coincident diagram that corresponds to the square torus 


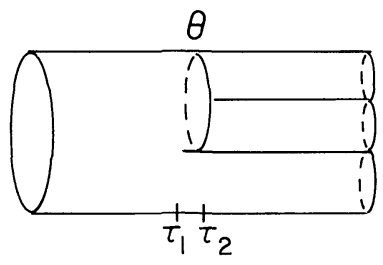

(a)

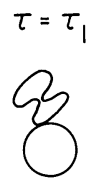

(b)

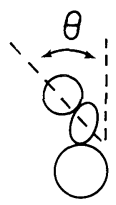

(c)

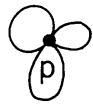

(d)

Fig. 4a-d. The limit as two interaction times become equal, but with a non-zero twist angle. In a is shown the world sheet with small time separation and non-zero twist; $\mathbf{b}$ shows the corresponding string configuration at time $\tau_{1}$. In $\mathbf{c}$ we see the interaction corresponding to the limit $\tau_{2}=\tau_{1}$, and in d we see the configuration resulting from equal interaction times and twist angle $\theta=\pi$

In this example the point on the world-sheet corresponding to identified points $a, c$, and $e$ is the site of a four string interaction (one incoming, three outgoing). One way to see how this interaction arises is to consider the limiting case when two interaction times become equal but the interaction points remain separated, as in Fig. 4. If the twist angle of the intermediate string is held fixed and the two times are allowed to become equal, the string configuration at the interaction time appears as shown in Fig. 4c. If the twist angle is taken to be $\theta=\pi$, we get the configuration in Fig. $4 \mathrm{~d}$. Here the point $p$ corresponds to the points $a, c, e$. In this interaction one strings splits into three strings. Clearly there are also configurations corresponding to higher order interactions as well. At first sight we might conclude that these pictures correspond to new interaction terms in the light-cone string theory, but this is not so because the scattering diagrams obtained from such configurations correspond to domains of measure zero in the moduli space. Another way of saying this is that the apparently new interactions in Fig. $4 \mathrm{c}$ and $\mathrm{d}$ are just the limiting cases of multiple simple interactions (Fig. 2), where the interaction times become equal.

Example 3. (Non trivial combinatorics) the reader will now recognize that the schematic (Fig. 3c) indeed gives a light-cone diagram.

Example 4. (Time coincident) consider Fig. 3d. Similar to the preceding examples define $\quad z_{a}=(w-a)^{1 / 2}, \quad \frac{\pi}{4}<\arg \left(z_{a}\right) \leqq \frac{3 \pi}{4}, \quad z_{c}=(w-c)^{1 / 2}, \quad \frac{3 \pi}{4}<\arg \left(z_{c}\right) \leqq \frac{5 \pi}{4}$, $z_{b}=(w-b)^{1 / 2}, \frac{5 \pi}{4}<\arg \left(z_{b}\right) \leqq \frac{7 \pi}{4}, z_{d}=(w-d)^{1 / 2}, \frac{7 \pi}{4}<\arg \left(z_{d}\right) \leqq \frac{9 \pi}{4}$, where these four charts combine to give a coordinate $z$ at the point represented by identifying $a$, $b, c$, and $d$. A similar identification is made for the other vertex. As before $2 z_{*} d z_{*}=d w$ and again $d w$ is the restriction to the world sheet of a meromorphic differential on the diagram. An amusing point is that we actually know this Riemann surface. By computing the Euler characteristic $F-E+V$, we check that it has genus one. If the height of the strip is $2 \pi i$ then the translation $w \rightarrow w+\frac{\pi i}{2}$ is compatible with the identifications and fixes the points $\tau=-\infty, \tau=+\infty$. The 
translation determines an order four symmetry $\varrho$ of the torus with two fixed points. An order four symmetry is unique to the torus $C /\{1, i\}$ and has precisely two fixed points, represented by 0 and $\frac{1+i}{2}$. S. Mandelstam was already aware of such time coincident diagrams [2].

From these examples we see by construction that the light-cone diagrams always define a Riemann surface with abelian differential. Conversely, given an abelian differential $\omega$ on an arbitrary Riemann surface, we can use that differential to define a flat structure with isolated singularities. To do this we define the coordinate $w$ in a neighborhood excluding a pole or zero of $\omega$ by the relation $w=\int_{z_{0}}^{z} \omega$; here $z$ is a local coordinate on the Riemann surface and $z_{0}$ is some basepoint in the neighborhood. Then $d w=\omega$ and an almost globally flat metric on the surface is $d w d \bar{w}=|\omega|^{2}$. However, this definition of $w$ breaks down at the zeroes and poles of the Abelian differential $\omega$. Consider for example simple zeroes or simple poles. At a simple zero $Z_{0}$, we have $d w \sim\left(z-Z_{0}\right) d z$ or $\left(w-W_{0}\right) \sim\left(z-Z_{0}\right)^{2}$. Therefore as we travel around the point $Z_{0}$ in the $z$ coordinate, in the process sweeping out an angle of $2 \pi$, we sweep out an angle $4 \pi$ in the $w$ coordinate about $W_{0}$. It is easy to see that this corresponds precisely to a simple interaction point in the light-cone picture labelled by the coordinate $w$. (Higher order zeroes just correspond to the higher order interactions mentioned above.) Similarly at a pole, we have $d w \sim \frac{d z}{z-Z_{0}}$ or $w \sim \ln \left(z-Z_{0}\right)$; this takes a small disk whose center is the point $Z_{0}$ to a tube propagating off to infinity in the $w$ coordinate. This clearly

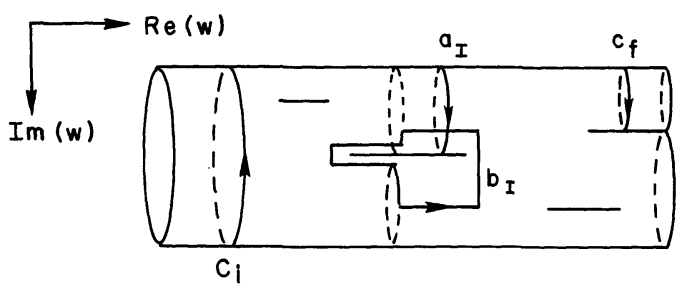

(a)

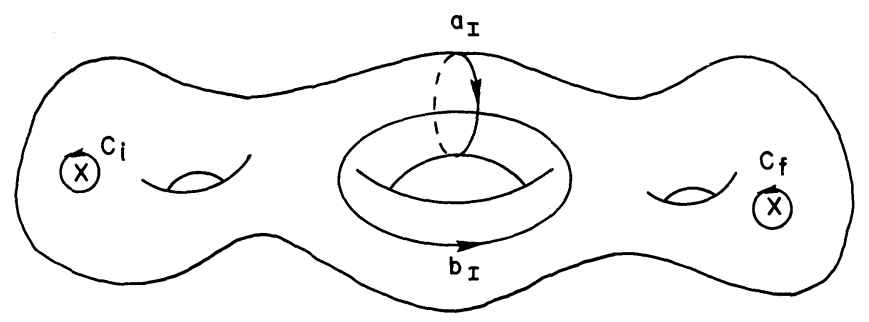

(b)

Fig. 5a and b. a The world sheet with local coordinate $w . \operatorname{Re}(w)$ increases in the direction of increasing light-cone time. Note that because of the twists the cycle $b_{I}$ doesn't close unless we add vertical segments. b A Riemann surface in the same conformal class as the light-cone diagram in a, with corresponding curves shown 
corresponds to an incoming or outgoing string in the light-cone picture, depending on the sign of the residue at the pole.

We can say even more about the abelian differential that provides the flat structure on the world sheet. We take the orientation to be as shown in Fig. 5a with the real part of $w$ increasing in the direction of increasing light-cone time and the imaginary part of $w$ increasing along the strings. Then if we integrate $d w$ along the contour $C_{i}$ around an incoming state as shown in Fig. 5a, we get for the integral $-2 \pi i \alpha_{i}$, where $2 \pi \alpha_{i}$ is the circumference, i.e. incoming momentum $2 \pi P_{i}^{+}$of the external state. On the conformally equivalent surface shown in Fig. 5b, however, we are integrating $\omega$ along the corresponding contour $C_{i}^{\prime}$, so we see that $\omega$ must have a simple pole of residue $-\alpha_{i}$ at the corresponding point $P_{i}$. Similarly if we integrate along the contour $C_{f}$ we get $2 \pi i$ times $\alpha_{f}$ for the final state, so $\omega$ must have a simple pole of residue $\alpha_{f}$ at the point $P_{f}$. Notice that from the geometry of the light-cone diagram we easily check that the sum of the residues at the poles is zero. We can also integrate around an internal tube. For example, if we integrate along the contour $a_{I}$ we get the answer $2 \pi i \alpha_{I}$, so the Abelian differential $\omega$ must satisfy $\oint_{a_{I}} \omega=2 \pi i \alpha_{I}$. In other words $\omega$ has period $2 \pi i \alpha_{I}$ along the cycle $a_{I}$. We can alternately look at the integral along the cycle $b_{I}$ (formed from horizontal and vertical arcs) as shown in Fig. 5a and b; because of the twists of the internal string if we follow lines of constant $\operatorname{Im}(w)$ the contour doesn't close. We must add a segment along a constant $\operatorname{Re}(w)$ contour (as shown) to close the contour ${ }^{3}$. The real part of the integral is zero since we get equal and opposite contributions to $\Delta \operatorname{Re}(w)$ from the top and bottom parts of the contour, so the period $\oint_{b_{I}} \omega$ is also pure imaginary ${ }^{4}$.

So far our discussion has shown that corresponding to the light-cone diagram there is an abelian differential $\omega$ with simple poles such that the residues at the poles are real and sum to zero. Further, the periods of the differential $\omega$ are pure imaginary. Suppose conversely that we are given a Riemann surface $R$ with specified points $P_{i}, i=1, \ldots, n$. Furthermore suppose we are given $n$ real numbers $\alpha_{i}$ such that $\sum_{i=1}^{n} \alpha_{i}=0$. Then there exists a unique meromorphic abelian differential $\omega$ with pure imaginary periods ${ }^{5}$, and whose singularities consist of simple poles at the points $P_{i}$ with residues $\alpha_{i}$. To show this, first consider the $g$ dimensional space of holomorphic abelian differentials. Given a homology basis as shown in Fig. 6, there exists a basis $\omega_{I}, I=1, \ldots, g$ for the abelian differentials such that $\oint_{a_{I}} \omega_{J}=\delta_{I J}$. The period matrix $\Omega_{I J}$ is given by $\Omega_{I J}=\oint_{b_{I}} \omega_{J}$. Furthermore, given two points $P$

\footnotetext{
${ }^{3}$ We define the twist angles to be positive in the right-handed sense for increasing light-cone time; then $\oint_{b_{I}} \omega=\frac{i}{2 \pi}\left(\alpha_{1} \theta_{1}-\alpha_{2} \theta_{2}\right)$

${ }^{4}$ If we wanted the light-cone construction for the open string we just set all the twists (including the integrals $\oint_{b_{I}}(\omega)$ to zero and then cut the world sheet in half to obtain the open string light-cone diagram

${ }^{5}$ Such differentials have been previously studied; see e.g. [7]. Further, S. Mandelstam has constructed the integrated expression $w$ for such differentials in the Schottky picture of a general Riemann surface $\lceil 8\rceil$
} 


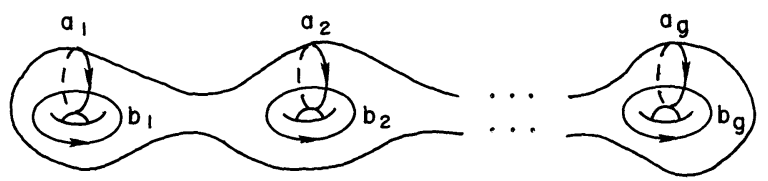

Fig. 6. The choice of a canonical homology basis on a general Riemann surface

and $Q$ on the Riemann surface $R$ there exists a meromorphic differential $\mu_{P Q}$ whose singularities are simple poles of equal and opposite residues at the points $P, Q$; for simplicity we take these residues to be $+1,-1$ respectively [9]. The space of meromorphic differentials whose singularities are simple poles of opposite residues at the points $P, Q$ is $g+1$ dimensional. This can be seen from Riemann-Roch or alternately by considering a genus $g+1$ surface on which we have pinched off one handle to obtain the genus $g$ surface with nodes at points $P$, $Q$; we can pick the $g+1$ basis holomorphic differentials on the higher genus surface so that $g$ of them remain holomorphic after pinching, and one develops simple poles ${ }^{6}$. Therefore the most general abelian differential with singularity set consisting of simple poles at the points $P_{1}, \ldots, P_{n}$ with the specified (real) residues $\alpha_{1}, \ldots, \alpha_{n}$ is

$$
\omega=\mu+\sum_{I=1}^{g} E_{I} \omega_{I},
$$

where to define $\mu$ we choose an arbitrary point $Q$ and set $\mu=\sum_{i} \alpha_{i} \mu_{P_{i} Q}$, and where $E_{I}=G_{I}+i F_{I}$ are arbitrary complex coefficients. Let the periods of $\mu$ be given by ${ }^{7}$ $\oint_{a_{I}} \mu=C_{I}+i A_{I}, \oint_{b_{I}} \mu=D_{I}+i B_{I}$. Then the periods of $\omega$ are

$$
\begin{aligned}
& \oint_{a_{I}} \omega=\left(C_{I}+G_{I}\right)+i\left(A_{I}+F_{I}\right), \\
& \oint_{b_{I}} \omega=\left[D_{I}+\sum_{J}\left(\Phi_{I J} G_{J}-\Psi_{I J} F_{J}\right)\right]+i\left[B_{I}+\sum_{J}\left(\Psi_{I J} G_{J}+\Phi_{I J} F_{J}\right)\right],
\end{aligned}
$$

where we have written the period matrix in terms of its real and imaginary parts, $\Omega_{I J}=\Phi_{I J}+i \Psi_{I J}$. The requirement of pure imaginary periods then specifies a unique solution for the constants $F_{I}, G_{I}$. This is given by

$$
G_{I}=-C_{I}, F_{I}=\sum_{J}\left(\Psi^{-1}\right)_{I J}\left[D_{J}+\sum_{K}\left(\Phi_{J K} G_{K}\right)\right] .
$$

Notice that to write the solution we had to invert the imaginary part of the period matrix, which is always possible since the imaginary part is positive definite. This construction therefore gives the unique differential $\omega$ with the specified pole structure and pure imaginary periods. Note that the differential is invariant (intrinsic) in the following sense: given a surface $R$ (respectively $\widetilde{R}$ ) with points

\footnotetext{
${ }^{6}$ If a cycle $A$ is to be pinched choose $\omega_{I}, 1 \leqq I \leqq g$, such that $\oint_{A} \omega_{I}=0$ and $\omega_{*}$ such that $\oint_{A} \omega_{*}=2 \pi i$

${ }^{7}$ Note that these periods are defined only modulo the contour integrals at the poles. To eliminate this ambiguity, when choosing the $a_{I}$ and $b_{I}$ cycles we assume their homotopy classes to be specified with respect to the surface with the points $P_{i}$ removed
} 
$P_{1}, \ldots, P_{n}$ (respectively $\widetilde{P}_{1}, \ldots, \widetilde{P}_{n}$ ) and residues $\alpha_{1}, \ldots, \alpha_{n}$, then for $f$ a conformal equivalence, $f: R \rightarrow \widetilde{R}, f\left(P_{j}\right)=\widetilde{P}_{j}$ indeed $\omega_{\tilde{R}^{\circ}} f=\omega_{R}$.

The choice of all imaginary periods is crucial for invariance under the mapping class group. If we had taken, say, a certain set of homology cycles and specified their periods, we would not expect a mapping class group invariant assignment. This is because we would have chosen some specific cycles, and the mapping class group mixes the cycles. However, for all imaginary periods, the construction is invariant under the mapping class group-there is no choice of any particular cycles. Thus $\omega$ depends only on the conformal structure of the surface.

One might ask whether a similar uniqueness theorem holds for differentials without poles, allowing us to put a similar structure on the closed Riemann surfaces, i.e. those without external states. We could imagine looking for the unique differential $\sum_{I} E_{I} \omega_{I}$ with pure imaginary periods. However, this doesn't work: for all the periods to be imaginary and the differential nontrivial, we find that $\operatorname{det}\left(\operatorname{Im} \Omega_{I J}\right)=0$, which corresponds to a degenerate Riemann surface. Therefore we are back where we started, with one handle pinched off to form two punctures. So as in the case of the triangulation of moduli space given by the open string field theory, we still cannot treat closed Riemann surfaces.

We now have the unique abelian differential $\omega$, but we are not quite done because we also must show that to each such differential there corresponds a lightcone diagram. To see what could possibly go wrong, recall that if we have a surface consisting of flat tubes glued together as in Fig. 5a, we have the structure of a family of closed vertical curves and the orthogonal family $\operatorname{Im}(\omega)=0$. These vertical curves correspond to the string or strings at a fixed light-cone time [i.e. fixed $\operatorname{Re}(w)$ ], and will be called the imaginary trajectories of the differential $\omega$. The problem is that the imaginary trajectories of an abelian differential don't always close; they can exhibit an ergodic type behavior. A simple example is the differential $\omega=e^{i \varphi} d z$ on the ordinary torus represented as a square with opposite sides identified; if the angle $\varphi$ specifies a line with irrational slope the imaginary trajectories of $\omega$ never close. This behavior would destroy the nice picture of the Riemann surface as constructed from flat tubes with a globally defined light-cone time. To show that this can't happen, notice that in the case of the torus, given a point on a trajectory, the trajectory itself will return an infinite number of times to an arbitrary neighborhood of this point. This is in fact the generic behavior for a differential on a compact Riemann surface: if a trajectory of an abelian differential does not terminate at a zero or pole and does not close, then it will necessarily have a limit cycle. By contrast, in our case, if there were such a trajectory we could follow it from a point forward to another point in a small neighborhood of the original point; then the integral $\int \omega$ along this trajectory segment has vanishing real part. We could of course take the two points to lie in a neighborhood excluding the zeroes and poles of $\omega$. Since we know what the trajectory structure looks like locally (see Fig. 7), we see that we could then close the trajectory by adding a small piece of an orthogonal trajectory along which $\operatorname{Im}(\omega)=0$. But over this piece $\operatorname{Re}(\omega) \neq 0$, so consequently the integral along the resultant closed contour would have non-zero real part. But this is clearly impossible since $\omega$ has pure imaginary periods. Therefore the imaginary trajectories of $\omega$ do indeed close. 


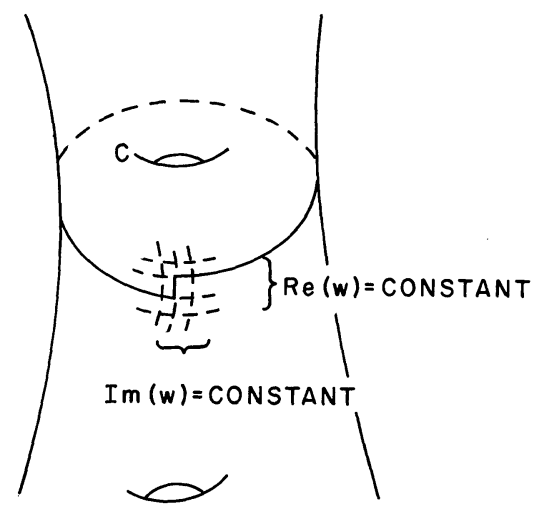

Fig. 7. The local trajectory structure away from a zero or pole of $\omega$, together with a section of a non-closed trajectory

A key feature of the light-cone gauge is the existence of a globally defined time (light-cone time), $\tau(z)=\operatorname{Re} \int_{z_{0}}^{z} \omega, \omega\left(z_{0}\right) \neq 0$; in fact $\tau(z)$ is reminiscent of the height functions of Morse theory. To this end define a critical value of $c$ of the time $\tau$ to be a real number such that $\omega$ vanishes somewhere on $\tau^{-1}(c)$ and the critical set is $\tau^{-1}(\{$ critical values $\})$. As with a Morse function the behavior of $\tau$ near a critical point is easily classified. If $p$ is a critical point then $\tau(z)=\operatorname{Re}\left(\int_{p}^{z} \omega+\int_{z_{0}}^{p} \omega\right)=\operatorname{Re}\left(z^{n}\right)+$ const, where $\omega$ is $n z^{n-1} d z$ in a local coordinate $z$ with $z(p)=0$. And of course the complement of the critical set is a union of $\omega$ cylinders (surfaces with differential, obtained by gluing $d w$ on a horizontal rectangle).

To see this consider an interval $[a, b]$ free of critical values. Then $\omega$ is zero free on $C=\tau^{-1}([a, b])$ and $d s^{2}=|\omega|^{2}$ defines a metric. In the light-cone picture local coordinates $w=x+i y, \omega=d w, \tau=x+$ const and $\operatorname{grad} \tau=\frac{\partial}{\partial x}$. The nonzero vector field grad $\tau$ defines a local flow which caries a level set $\tau^{-1}(\alpha), a<\alpha<b$ to $\tau^{-1}(b)$. C is the product of the interval $[a, b]$ and a disjoint union of circles. The full conclusion follows since $d \tau=\operatorname{Re}(\omega)$.

In summary, we have seen that for each conformal structure on a Riemann surface and each specification of points and of residues (i.e. external $P^{+}$momenta) at these points we have a unique light-cone diagram. The light-cone diagrams cover moduli space, and the parameters $(\alpha, \tau, \theta)$ act as a global set of coordinates. We have a new cell decomposition of moduli space.

The top dimensional cell appears with some boundary identifications as the set $\mathscr{S}$ of simple diagrams ( $\omega$ has simple zeroes). $\mathscr{S}$ is in fact connected or equivalently any simple diagram can be deformed continuously through simple diagrams to the normal form of Fig. 8. To see this we introduce the notions of splits and joins; keep in mind that the time axis is oriented and the following is for the simple diagrams of $\mathscr{S}$. There are four sectors near a simple interaction point $p$ : if $\tau_{0}$ is the interaction 


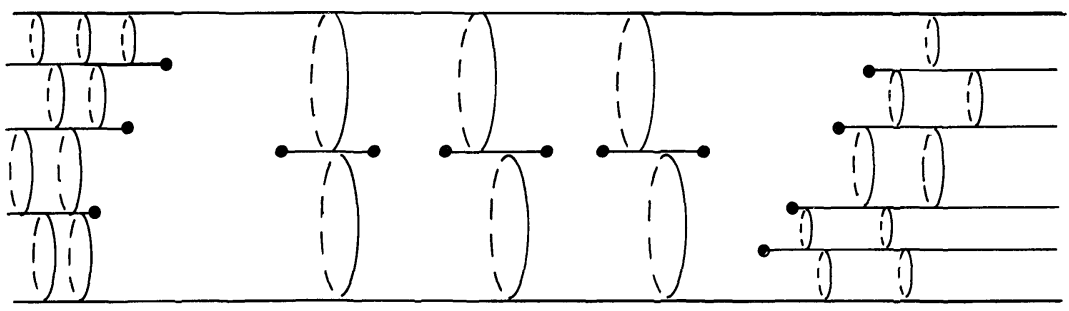

Fig. 8. The normal form for a genus 3,4 incoming and 5 outgoing diagram

time then each time interval $\left(\tau_{0}-\varepsilon, \tau_{0}\right),\left(\tau_{0}, \tau_{0}+\varepsilon\right)$ determines two regions near $p$. A string separates into two at a split. A split $p$ is rejoined at the interaction $q$ (not necessarily unique) provided that there exist paths $\gamma_{1}, \gamma_{2}$ such that: $\gamma_{1}(0)=\gamma_{2}(0)=p$, $\gamma_{1}(1)=\gamma_{2}(1)=q$, time is strictly increasing along $\gamma_{1}, \gamma_{2}$ and the paths leave $p$ in distinct sectors. A split $p$ is internal if it is rejoined at some interaction $q$; otherwise it is outgoing. Joins, internal joins, and incoming joins are defined by reversing the time axis. Finally there are two forward and two backward real trajectories, the interaction signal, emanating from an interaction point.

The basic situation is for a split $p$ and a joint $q$ with $\tau(p)<\tau(q)$ (and no intermediate interactions) and $p$ does not rejoin at $q$. A small perturbation of the diagram will insure that the $p$ and $q$ signals are distinct for each time slice. The two forward $p$ signals pick out two components of a $\tau_{1}$ time slice, $\tau(p)<\tau_{1}<\tau(q)$, and similarly for the two backward $q$ signals. Since $p$ does not rejoin at $q$ there must be at least three components (the maximum for two interactions) and the diagram is given by Fig. 9a. Simply push the join $q$ to the left of $p$ to obtain the diagram Fig. 9b.

Now consider the general diagram in $\mathscr{S}$ : genus $g, m$ incoming strings, $n$ outgoing strings. By a small perturbation we arrange that all interaction times are distinct and all interaction signals are distinct for each time slice. The discussion for the basic situation insures that each outgoing split may be pushed, through diagrams in $\mathscr{S}$, to the far right; and each incoming join pushed to the far left. Thus it is enough to consider diagrams with one incoming and one outgoing string. For such a diagram consider the (internal) split $p$ with the largest time value. This situation is special: $p$ is rejoined at a unique join $q$. In fact if $\gamma$ is a path leaving $p$, for which time is strictly increasing, then its topology (homotopy class relative to the terminal time slice) is completely determined by its sector leaving $p$. The reason: there are no splits to the right of $p$ and so no decisions for $\gamma$. The consequence: if $p$ is rejoined at $q$ then the topology of the joining paths (one for each sector) is completely determined; $q$ is unique. The conclusion: every other join to the right of $p$ can be pushed to the left of $p$. Now proceed by induction to move to the normal form.

The parametrization of this cell is not completely trivial in terms of the coordinates $(\alpha, \theta, \tau)$. The reason for this is that we will overcount configurations if we allow $(\alpha, \theta, \tau)$ to range freely - this comes from the fact that the handles in the surface are indistinguishable, yet we have labelled them while introducing coordinates. For example in the simple case of Fig. 10 the naive count gives two 


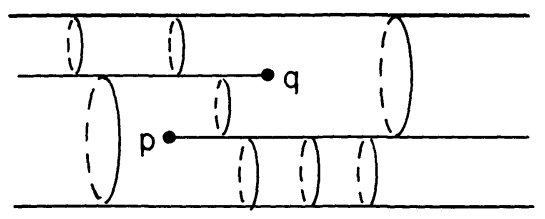

(a)

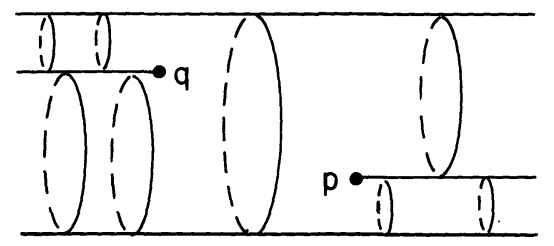

(b)

Fig. 9a and b. In $\mathbf{a}$ is a split $p$ which is not rejoined at $q$, and $\mathbf{b}$ shows the result of pushing $q$ past $p$
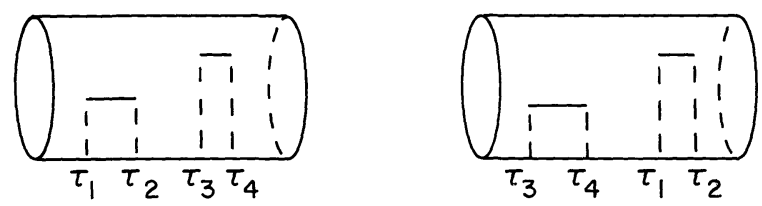

Fig. 10. An example of the overcounting problem. These two diagrams arise from different ranges of the parameters $\tau_{1}, \ldots, \tau_{4}$, but are clearly geometrically identical

diagrams even though the actual count is one. This overcounting problem is similar to the one encountered in $[3,5,6]$ and should not be difficult to solve; we must simply divide by discrete symmetry factors. These are reminiscient of the combinatorical symmetry factors that one encounters in ordinary Feynman diagrams.

An appealing aspect of this cell decomposition is that the coordinates appear to be closely related to the complex structure of moduli space. One reason for this is that the coordinates correspond to periods of an abelian differential or to the integrals of that differential between its zeroes. The expressions for the periods are especially easy to write down using Riemann's bilinear relations; for the case of a differential $\omega_{P_{1} P_{2}}$ with residues $+1,-1$ at points $P_{1}, P_{2}$ and pure imaginary periods we have (see e.g. [7])

$$
\oint_{b_{I}} \omega_{P_{1} P_{2}}-\sum_{J=1}^{g} \Omega_{I J} \oint_{a_{I}} \omega_{P_{1} P_{2}}=2 \pi i \int_{P_{2}}^{P_{1}} \omega_{I} .
$$

This then gives all of the internal momenta $\alpha_{I}$ and combinations of twist angles $\theta_{\beta}$ for each internal loop. These are expressed directly in terms of the period matrix and the integrals of the holomorphic differentials between the specified points, so the relations are simple in the complex parameters on moduli space. For example, 
in the case of the torus the complex modular parameters are the usual $\tau$ (the torus is the quotient of $C$ by the lattice $\{1, \tau\}$ ) together with the coordinates of the points corresponding to the external states, $Z_{1}, Z_{2}$. The relation between these and lightcone parameters is given by

$$
2 \pi i\left(Z_{1}-Z_{2}\right)=\left(\theta_{1}+\theta_{2}\right) \alpha_{1}-\theta_{2}-2 \pi i \alpha_{1} \tau .
$$

Of course to solve for the individual twist angles and the interaction times we must know the integrals between the zeros of $\omega$. In the case of the torus these integrals are given by theta functions in the variables $\tau, Z_{1}, Z_{2}$, so once again we have variables locally analytic in the complex parameters on moduli space. $\mathrm{S}$. Mandelstam has already noted that the abelian integral $w=\int^{z} \omega$ can be written explicitly in terms of theta functions [10]. In particular,

$$
w=\ln \theta\left[\begin{array}{l}
1 \\
1
\end{array}\right]\left(z-\zeta_{1}, \tau\right)-\ln \theta\left[\begin{array}{l}
1 \\
1
\end{array}\right]\left(z-\zeta_{2}, \tau\right)+2 \pi i z \frac{\operatorname{Re}\left(2 \pi i\left(\zeta_{2}-\zeta_{1}\right)\right)}{\operatorname{Re}(2 \pi i \tau)},
$$

where $\frac{d w}{d z}$ has simple zeroes at $\zeta_{1}$ and $\zeta_{2}$ (see [11]). This feature should extend to higher genus: $2 g$ relations determining the parameters are given by the simple expression (2), and the remaining $4 g+2 n-6$ relations should be given in terms of the higher genus analogues of the simple genus-one theta functions. Consequently there are likely simple analytic expressions relating the light-cone parameters and the complex coordinates on moduli space.

Although previously physicists may have thought of the light-cone formalism as inelegant, we see now that the formalism is extremely natural from the point of view of the complex structure of the Riemann surface. We also see an obvious way to change the gauge, i.e. the choice of external $P^{+}$momenta, in the light-cone formalism: we just choose different $\alpha_{i}$ coefficients for our differentials $\mu_{P_{i} Q}$. Furthermore, these results lend credence to the idea of trying to use the light-cone picture of interactions to formulate a string field theory for the closed string ${ }^{[12]}$. Although the light-cone formalism itself appears to depend on choice of gauge, the scattering diagrams it produces really have a deep and natural connection to the underlying Riemann surface. Therefore it is not unreasonable to think that the closed string field theory might have a construction whose basis is the light-cone type Feynman diagram. Notice that we also have the added bonus of a construction that closely captures the complex structure on moduli space; this could be important for the formulation of string theories like the heterotic string.

One should also note that the proof, that the light-cone diagrams give a one-toone cover of moduli space, is one part of the proof of the equivalence between the light-cone/interacting string picture and the Polyakov approach. We have shown that both formalisms give the same integration region (namely moduli space); what remains to be shown is that they give the same integration measure. Of course this is one method that could be used to prove that the Polyakov theory is unitary, since in the light cone formalism unitary is manifest.

In conclusion, we see that the light-cone scattering diagrams of string theory cover moduli space and provide a very nice parametrization of that space. The parametrization for generic surfaces is extremely simple. This fact is relevant for 
the integration over moduli space since exceptional surfaces form sets of codimension one or higher. Nevertheless, since in essence we are representing moduli space as one cell, the identifications on the boundary must be relatively complicated in order to agree with the known topological complexity of moduli space.

Acknowledgements. The authors would particularly like to thank S. Mandelstam for sharing his ideas on constructing light-cone diagrams for the general Riemann surface, and E. Witten for helpful comments, including one on the overcounting problem. One of us (SBG) would also like to thank C. Poor for pointing out to him some relevant facts regarding abelian differentials.

\section{References}

1. Polyakov, A.M.: Quantum geometry of bosonic strings, and quantum geometry of fermionic strings. Phys. Lett. 103B, 207, 211 (1981)

2. Mandelstam, S.: Interacting-string picture of dual-resonance models. Nucl. Phys. B64, 205 (1973); Dual-resonance models. Phys. Rep. 13C, 259 (1974); see also Mandelstam, S.: In: Unified string theories. Proceeding of the Santa Barbara workshop on Unified String Theories. Green, M., Gross, D. (eds.). New York: World Scientific

3. Harer, J., Zagier, D.: The Euler characteristic of the moduli space of curves. Maryland Math. preprint (April 1985)

4. Witten, E.: Non-commutative geometry and string field theory. Nucl. Phys. B268, 253 (1986)

5. This cell decomposition is based on an idea due to $\mathrm{W}$. Thurston (unpublished), with details provided by Bowditch and Epstein; the first complete proof was by D. Mumford (unpublished) and J. Harer. See Harer, J.: The virtual cohomological dimension of the mapping class group of an orientable surface. Maryland Math. preprint (July 1984) or Harer, J.: The cohomology of the moduli space of curves, C.I.M.E. Lecture Notes

6. Giddings, S., Martinec, E., Witten, E.: Modular invariance in string field theory. Phys. Lett. 176B, 362 (1986)

7. Farkas, H., Kra, I.: Riemann surfaces. Berlin, Heidelberg, New York: Springer 1980

8. Mandelstam, S.: Private communication

9. Gunning, R.C.: Lectures on Riemann surfaces. Princeton, W: Princeton University Press 1966

10. Mandelstam, S.: In: Unified string theories, ref. [2]

11. Lebowitz, A., Rauch, H.: Elliptic functions, theta functions, and Riemann surfaces. Baltimore, MD: Williams and Wilkins 1973, pp. 74-81

12. Some progress in this direction has been made by Hata, H., Itoh, K., Kugo, T., Kunitomo, H., Ogawa, K.: Kyoto University preprints (December 1985, March 1986); The gauge-covariant formulation of interacting strings and superstrings. Awada, M.A.: Phys. Lett. 172 B, 32 (1986) and Cambridge preprints (January, February, and September 1986)

Neveu, A., West, P.: The interacting gauge covariant bosonic string, Phys. Lett 168B, 192 (1986) and CERN preprints (1986)

13. D'Hoker, E., Giddings, S.: In preparation

Communicated by A. Jaffe

Received November 10, 1986

Note added in proof. The interaction signals provide a natural decomposition of the surface into bi-infinite strips; the light cone structure restricted to such a strip makes it Euclidean. Generally there are two interaction points per strip, and more in the case of non-generic surfaces. The combinatorics of these strips encodes the cell decomposition of moduli space.

Recently [13] the Polyakov integration measure has been shown equivalent to that of the light-cone formalism, establishing the equivalence of the two approaches. 